PROFESIONALES Y HERRAMIENTAS PARA EL DESARROLLO LOCAL Y SUS SINERGIAS TERRITORIALES. EVALUACIÓN Y PROPUESTAS DE FUTURO IX Coloquio Nacional de Desarrollo Local del GTDL-AGE 

ANTONIO MARTÍNEZ PUCHE, XAVIER AMAT MONTESINOS, ISABEL SANCHO CARBONELL y DANIEL SANCHIZ CASTAÑO (EDS.)

\section{PROFESIONALES Y HERRAMIENTAS PARA EL DESARROLLO LOCAL Y SUS SINERGIAS TERRITORIALES. EVALUACIÓN Y PROPUESTAS DE FUTURO}

IX Coloquio Nacional de Desarrollo Local del GTDL-AGE

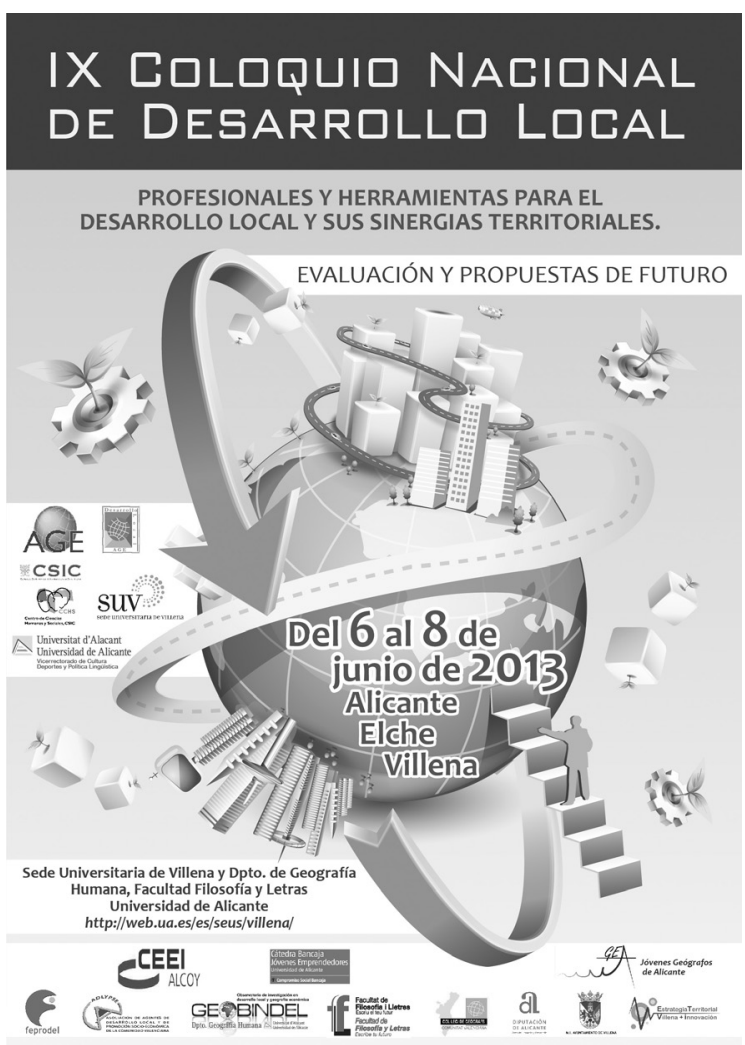


Este libro ha sido examinado y valorado por evaluadores ajenos a la Universidad de Alicante, con el fin de garantizar la calidad científica del mismo.

Publicacions de la Universitat d'Alacant

03690 Sant Vicent del Raspeig

Publicaciones@ua.es

http://publicaciones.ua.es

Telèfon: 965903480

(C) Antonio Martínez Puche, Xavier Amat Montesinos,

Isabel Sancho Carbonell y Daniel Sanchiz Castaño (eds.), 2016

(C) d'aquesta edició: Universitat d'Alacant

ISBN: 978-84-16724-00-0

Dipòsit legal: A 92-2016

Disseny de coberta: candela ink

Composició: Página Maestra (Miguel Ángel Sánchez Hernández)

Impressió i enquadernació: Guada Impresores

\section{unte \\ Unión de Editoriales
Universitarias Españolas \\ WWW.une.es
WWA}

Esta editorial es miembro de la UNE, cosa que garantiza la difusión y comercialización nacional y internacional de sus publicaciones.

Reservados todos los derechos. Cualquier forma de reproducción, distribución, comunicación pública o transformación de esta obra sólo puede ser realizada con la autorización de sus titulares, salvo excepción prevista por la ley. Diríjase a CEDRO (Centro Español de Derechos Repográficos, www.cedro.org) si necesita fotocopias o escanear algún fragmento de esta obra. 


\title{
LA CRISIS ECONÓMICA EN TERRITORIOS EN CRISIS ESTRUCTURAL. INSTRUMENTOS DE DESARROLLO EN LA CUENCA MINERA DE RIOTINTO
}

\author{
Francisco Cabello López \\ Museo Minero de Riotinto Fundación de Riotinto \\ museoriotinto@telefonica.net \\ Francisco Javier García Delgado \\ Museo Minero de Riotinto Fundación de Riotinto \\ fcogarci.uhu@gmail.com \\ Aquilino Delgado Domínguez. \\ Museo Minero de Riotinto Fundación de Riotinto \\ museomineroriotinto@telefonica.net
}

\section{RESUMEN}

El cese de la actividad minera (monocultivo económico) en áreas especializadas condujo a una crisis estructural a territorio hasta entonces dinámicos (por la generación de empleo y renta), aunque abocados a un modelo primario exportador. En este contexto de crisis permanente, comarcas como la Cuenca Minera de Riotinto fueron objetivo de programas y proyectos de desarrollo con la creación de una estructura y la implementación de instrumentos diversos por parte de las administraciones públicas y algunas iniciativas privadas. En el territorio confluyeron inversiones públicas (con fondos autonómicos, estatales y comunitarios), pero la dinamización territorial a través de las mismas ha tenido desigual fortuna en busca de la diversificación de actividades y la dinamización del territorio.

En la actualidad, el contexto de crisis económica mundial lleva al replanteamiento de las estructuras de desarrollo, que afectan al empleo y la inversión y ahondan en las desigualdades territoriales, la marginalidad y la marginación 
de la Cuenca Minera, al tiempo que el buen precio de los metales muestra la oportunidad de reabrir las minas.

El objetivo central de esta comunicación es analizar los efectos territoriales de la crisis económica en el desmontaje de las estructuras de desarrollo, el fin de la afluencia de fondos públicos y una valoración retrospectiva de la eficacia y eficiencia de los instrumentos de dinamización territorial empleados en la Comarca Minera de Riotinto durante los últimos decenios.

Palabras-clave: instrumentos y estructuras de desarrollo, Cuenca Minera de Riotinto, crisis económica, desarrollo local.

\section{ECONOMIC CRISIS IN TERRITORIES OF STRUCTURAL CRISIS. INSTRUMENTS OF DEVELOPMENT IN THE MINING BASIN RIOTINTO}

\section{ABSTRACT}

The cessation of mining activities (economic monoculture) in specialist areas led to a structural crisis in the territory that was previously dynamic (for the generation of employment and income), though doomed to a primary export model . In this context of permanent crisis regions such as the mining of Riotinto they were targeted programs and projects with the creation of a structure and the implementation of various instruments by public and some private initiatives. In the territory they converged public investments (with regional, national and EU funds), but the territorial revitalization through them has had unequal fortune in search of diversification of activities and the revitalization of the territory.

Today, the context of global economic crisis leads to rethinking structures development, affecting employment and investment and deepens the territorial inequalities, marginalization and exclusion of the mining, while good price metals shows the opportunity to reopen the mines.

The main objective of this paper is to analyze the regional effects of the economic crisis in the dismantling of the structures of development, to the influx of public funds and a retrospective assessment of the effectiveness and efficiency of the instruments used in territorial revitalization Rio Tinto mining region in recent decades.

Key words: instruments and structures development, Cuenca Minera Riotinto, economic crisis, local development.

\section{INTRODUCCIÓN}

En el último medio siglo se ha producido un cambio productivo en la mayor parte de las cuencas mineras europeas. Los procesos políticos (emancipaciones coloniales) y económicos de la segunda postguerra mundial condujeron al 
aumento de la competencia internacional de minerales producidos en Zaire, Chile, Suráfrica..., lo que unido a la baja rentabilidad de las minas europeas, menos productivas y con mano de obra más cara, y la bajada de los precios de los metales y el contexto de crisis energética de 1973 llevó a su cierre (GarcíaDelgado y otros, 2013).

De esta manera, los importantes y milenarios paisajes mineros perdieron su función extractiva/transformadora y la desindustrialización se convirtió en "un problema que iba en aumento y presentaba múltiples aristas: degradación medioambiental y física del espacio urbano, contaminación de suelos, especulación, destrucción de actividad económica, desempleo y tensión social" (Benito, 2002: 216), además de dificultar otras actividades económicas como la ganadería o la agricultura.

Desde la llegada de la democracia, y muy especialmente con la incorporación de España a la CEE, de la mano del FSE, los Fondos de Cohesión o las iniciativas comunitarias (LEADER, PRODER...), etc. se han buscado nuevas alternativas a la comarca, destinadas a:

- La dotación de servicios o el refuerzo de los ya existentes (sanitarios -Hospital Comarcal, único centro hospitalario del interior de la provincia-, centros educativos de secundaria).

- El establecimiento de una red de desarrollo (que nos ocupa).

- La formación (derivada de la anterior) a través de escuelas-taller y talleres de empleo y cursos diversos (Formación Profesional Ocupacional, ahora Formación Profesional para el Empleo), que permitan la inserción laboral.

- La recuperación del medio, especialmente con la restauración de parte del espacio para la nueva agricultura (plantaciones de cítricos y aguacates) fertirrigada.

- El fomento de la actividad industrial a través de la creación de polígonos industriales, estableciendo nuevas industrias (producción de plásticos, fertilizantes...).

- El fomento de la terciarización de la comarca a través del desarrollo turístico, vinculada al patrimonio minero y rural.

- La creación de un vertedero de residuos tóxicos y peligrosos en Nerva, retratándose una situación similar a la analizada en Cardona por Llurdés et al. (1999).

Sin embargo, como veremos, los resultados del modelo de desarrollo han sido desiguales.

\section{Caracterización de la Comarca de la Cuenca Minera de Riotinto}

La Comarca de la Cuenca Minera de Riotinto se sitúa en la zona centro-oriental de la provincia de Huelva, en el límite con la provincia de Sevilla. Está compuesta por siete municipios: El Berrocal, El Campillo, Campofrío, La Granada 
de Río Tinto, Minas de Riotinto, Nerva y Zalamea la Real, que ocupan una superficie total de $626,2 \mathrm{~km}^{2}$, el $6,18 \%$ del total provincial.

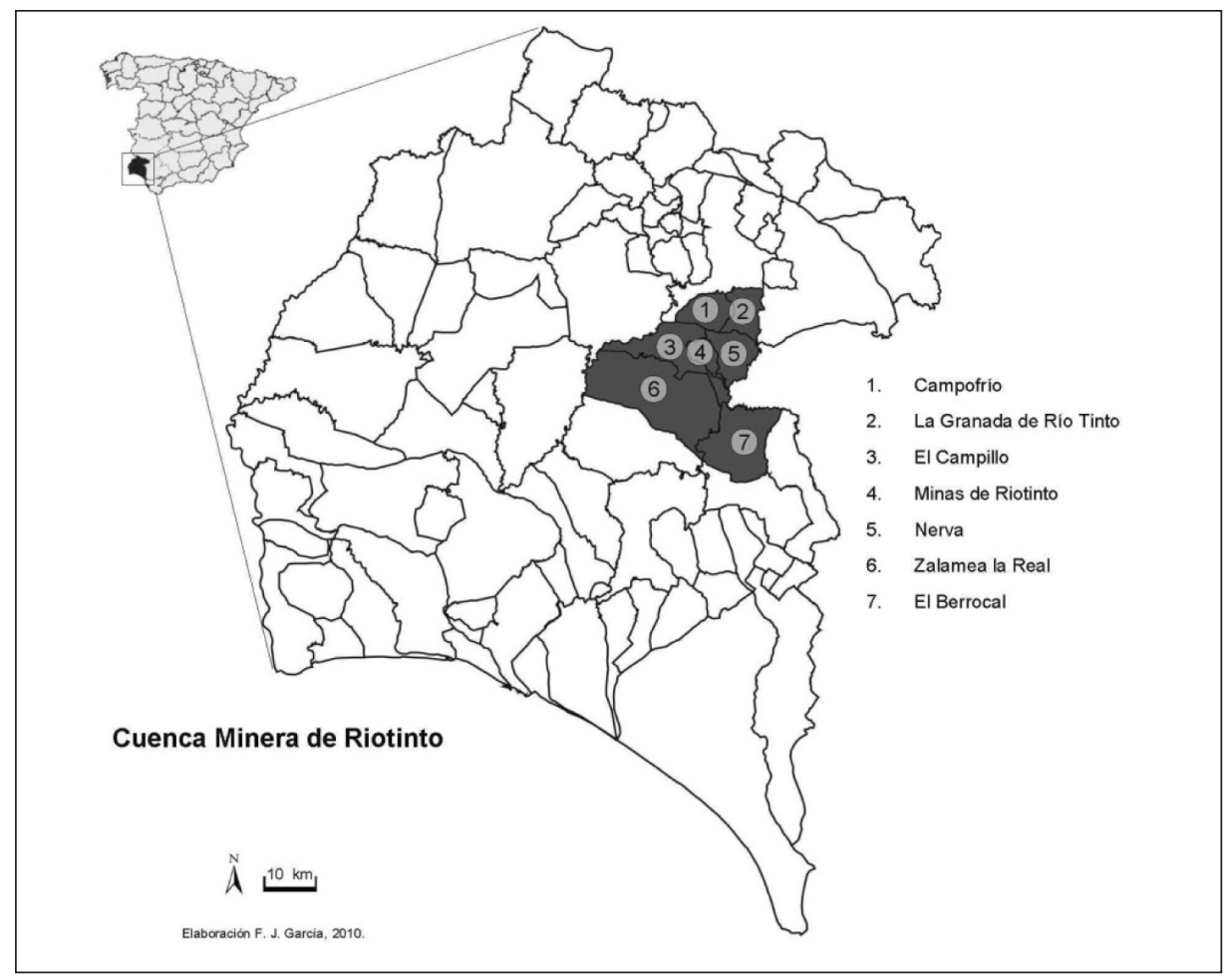

Mapa 1. La Cuenca Minera de Riotinto (Huelva).

Fuente: García-Delgado y otros, 2013; 134.

Mientras la zona central de la comarca se especializaba en la minería, las áreas sur (Zalamea la Real) y norte (La Granada de Río Tinto y Campofrío), a pesar de la existencia de minas, se especializaron en la agricultura, la explotación forestal y la ganadería para cubrir las demandas de las poblaciones mineras.

A partir de los años 50 del siglo XX, en el contexto de la nacionalización de las minas (1954), se inicia una profunda crisis por el aumento de los costes de producción y el retroceso en la competitividad por la aparición de nuevos competidores y nuevos minerales. Desde los años 60 se vive una relocalización de actividades hacia la capital provincial (Polos de Promoción -1964- y de Desarrollo -1968-) y la decadencia se acentúa con la crisis del 73. En 1986 se produce el cierre de la línea de cobre y en 1992 la crisis del mercado del oro lleva a una nueva regulación del empleo (Foronda y Joya, 2004: 294-295) y, desde ese momento, y hasta el cierre definitivo de las Minas de Riotinto, 


\begin{tabular}{|c|c|c|c|c|c|c|c|c|c|}
\hline 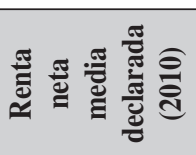 & $\begin{array}{l}\overrightarrow{0} \\
\stackrel{\infty}{\hat{0}} \\
0\end{array}$ & $\begin{array}{l}0 \\
+ \\
\infty \\
n \\
n \\
n\end{array}$ & $\begin{array}{l}\stackrel{0}{=} \\
\stackrel{\nabla}{\Xi}\end{array}$ & $\begin{array}{l}\infty \\
\infty \\
\stackrel{0}{+} \\
\stackrel{+}{a}\end{array}$ & $\begin{array}{l}\text { t. } \\
\text { \&ें } \\
\stackrel{0}{0}\end{array}$ & 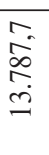 & $\begin{array}{l}\text { ๙ે̀ } \\
\text { ปे } \\
\dot{\Xi}\end{array}$ & 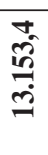 & 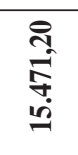 \\
\hline 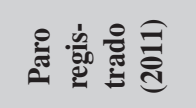 & $\approx$ & $\underset{\sim}{\stackrel{\sim}{N}}$ & $\cong$ & of & $\underset{⿱}{\infty}$ & $\begin{array}{l}\infty \\
\infty\end{array}$ & $\ddot{m}$ & 空 & ๙ூ \\
\hline 离 & $\stackrel{ }{\div}$ & $\underset{1}{ \pm}$ & $\mathcal{T}$ & $a$ & $\stackrel{\circ}{\bullet}$ & $n$ & $\stackrel{9}{1}$ & $\stackrel{7}{7}$ & त్తి \\
\hline 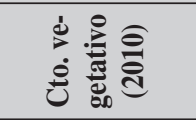 & $T$ & $\varphi_{1}$ & - & $?$ & 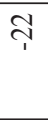 & $\stackrel{\infty}{+}$ & $\underset{1}{ \pm}$ & बे & ?ֶ: \\
\hline 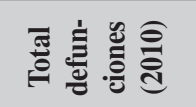 & $r$ & Әे & $r$ & $m$ & ஜิ & Ð & 우 & సิ & $\begin{array}{l}\stackrel{\partial}{\stackrel{\partial}{+}} \\
\text { }\end{array}$ \\
\hline 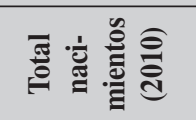 & 0 & $\ddot{\sim}$ & $\infty$ & 0 & $\hat{n}$ & r & $\stackrel{\sim}{\sim}$ & 户্লি & $\begin{array}{l}8 \\
\text { if } \\
\text { in }\end{array}$ \\
\hline 宓 & $?$ & $\infty_{1}$ & $?$ & $\simeq$ & $\stackrel{\sim}{\sim}$ & $\vec{i}$ & $n$ & 导 & $\mathscr{L}$ \\
\hline 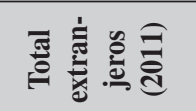 & $\sim$ & ஓ & ㄱ. & $m$ & $\mathscr{6}$ & $\stackrel{\infty}{\stackrel{\nu}{\nu}}$ & $\curvearrowleft$ & 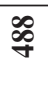 & તัँ \\
\hline 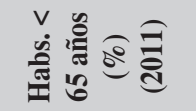 & $\begin{array}{c}\text { q } \\
\infty \\
\infty \\
\infty\end{array}$ & $\begin{array}{l}n \\
\stackrel{n}{8}\end{array}$ & $\begin{array}{l}\overrightarrow{0} \\
\triangleq\end{array}$ & $\begin{array}{l}\text { I } \\
\text { \& }\end{array}$ & $\overline{\text { i }}$ & $\begin{array}{c}\tilde{\lambda} \\
\tilde{\lambda}\end{array}$ & $\begin{array}{l}\stackrel{2}{2} \\
\tilde{\imath}\end{array}$ & స్ & 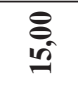 \\
\hline 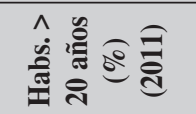 & ô. & $\stackrel{\cong}{\cong}$ & $=$ & $\begin{array}{l}\text { ñ } \\
\text { ô }\end{array}$ & $\begin{array}{l}\tilde{m} \\
\infty\end{array}$ & $\begin{array}{l}\underset{+}{J} \\
\infty\end{array}$ & \begin{tabular}{l}
\multirow{2}{n}{} \\
$\underline{0}$
\end{tabular} & $\begin{array}{l}\hat{S} \\
\text { ले }\end{array}$ & ते \\
\hline 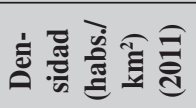 & $\underset{i}{\stackrel{i}{i}}$ & $\begin{array}{l}\stackrel{ \pm}{2} \\
\stackrel{+}{d}\end{array}$ & $\begin{array}{l}\text { an } \\
\text { I }\end{array}$ & $\begin{array}{c}\hat{n} \\
\text { ñ }\end{array}$ & 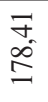 & 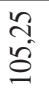 & $\begin{array}{l}\stackrel{n}{2} \\
\pm\end{array}$ & $\begin{array}{l}12 \\
8 \\
8\end{array}$ & $\underset{\text { 空 }}{\text { 至 }}$ \\
\hline 宓 : & gે & 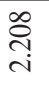 & $\bar{\varpi}$ & $\stackrel{\text { ㅇ }}{\text { O }}$ & $\frac{i n}{\underset{r}{*}}$ & $\begin{array}{l}\bar{\infty} \\
\infty \\
\dot{n}\end{array}$ & $\begin{array}{l}\stackrel{\infty}{\infty} \\
\text { m} \\
\text { m. }\end{array}$ & ڤั) & 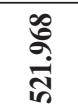 \\
\hline 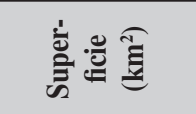 & $\begin{array}{l}\text { तิ } \\
\text { ป̂ } \\
\text { ป̂ }\end{array}$ & $\hat{8}$ & $\begin{array}{l}0 \\
\stackrel{+}{f}\end{array}$ & 柋 & $\hat{\tilde{\lambda}}$ & $\begin{array}{l}\text { In } \\
\text { in }\end{array}$ & $\begin{array}{l}\text { के } \\
\text { ते }\end{array}$ & స్రิ & $\stackrel{\mathscr{7}}{\stackrel{\Xi}{\Xi}}$ \\
\hline 爱 & 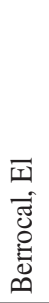 & 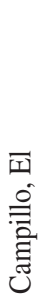 & 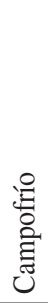 & 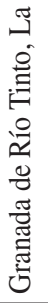 & 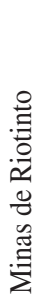 & 胥 & 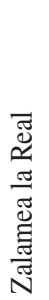 & 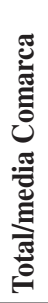 & 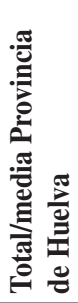 \\
\hline
\end{tabular}


en 2001, se conoce un progresivo descenso de la actividad económica y del empleo. A partir de entonces, una comarca sumida en el "paternalismo empresarial" de la Compañía comienza un nuevo camino como espacio improductivo, subsidiado y subvencionado.

La crisis de la actividad minera ha determinado la evolución demográfica de la comarca, que comenzó a sufrir una sangría poblacional desde los años sesenta y setenta del siglo XX por la litoralización de las actividades económicas (cfr. supra). En la actualidad supone una población reducida, que alcanza poco más del 3\% de los efectivos provinciales, con sus 16.976 habitantes (IEA, 2012), con unas características que señalan un futuro incierto: baja natalidad, envejecimiento, saldo migratorio negativo, altas tasas de dependencia..., muy similares a las de otras comarcas desindustrializadas (Valenzuela et al., 2008) y rurales (Márquez, 2002). Con respecto a la formación de la población, el desarrollo es escaso y reciente, y se producen dificultades de absorción de población altamente cualificada por el tejido productivo deficiente.

La renta media desciende, al tiempo que los jubilados y prejubilados de la mina van desapareciendo y los subsidios de desempleo se hacen fuertes en la comarca, y se plantean nuevas incertidumbres en el contexto de crisis.

\section{Las estructuras promotoras de desarrollo y empleo en la Cuenca Minera De Riotinto. Configuración actual, estado y funcionamiento}

La lucha contra el desempleo requiere de dispositivos y profesionales que, partiendo de un sólido conocimiento de sus respectivas dinámicas territoriales y de los diferentes programas y políticas activas de empleo, sean capaces de ofrecer soluciones a las necesidades y demandas específicas que les sean planteadas por las empresas y agentes de su entorno.

Los dispositivos y profesionales, en sus respectivos ámbitos locales de trabajo, deben aglutinar los intereses públicos y privados, en torno a estos nuevos retos, mediante su apoyo tanto al tejido productivo existente como al potencialmente naciente (iniciativas de autoempleo), contribuyendo a la creación de nuevos empleos, y liderando aquellos proyectos que pudieran ser susceptibles de generar nueva actividad económica y empleo (Cabello, 2011).

En torno a esta idea, en la Cuenca Minera de Riotinto, coexisten en la actualidad un conjunto de estructuras, que derivando de los programas y redes diseñadas por la administración autonómica y central, interactúan entre sí y con los beneficiarios, implementando sus programas, para conseguir sus objetivos. A continuación, vamos a relacionarlos, describiendo su configuración, funcionamiento y estado actual (Cabello, 2012). 


\subsection{Red de oficinas de empleo del Servicio Andaluz de Empleo (SAE)}

El Servicio Andaluz de Empleo (SAE) es creado por la LEY 4/2002, de 16 de diciembre, como instrumento público para la gestión de las políticas de empleo $\mathrm{y}$, con el tiempo, sustituye al INEM en el territorio andaluz. Así, al sustituir a este, se hace cargo de la oficina de empleo situada en Nerva, para atender al conjunto de la comarca.

Las actividades y acciones que realizan en la Cuenca Minera, son las que les vienen determinadas por los servicios centrales de la Consejería y/o la Delegación provincial. Entre sus funciones o acciones se encuentran:

- Actualización de datos y gestión de las ofertas que llegan a la oficina.

- Sellado de tarjetas de demanda de empleo y nuevas inscripciones.

- Vinculación de ofertas en difusión a demandantes inscritos.

- Emisión de certificados.

- Registro de contratos.

- Punto de empleo. Información a los ciudadanos de la Comarca de las ofertas laborales, de ofertas de cursos, red Eures, etc.

Los técnicos de esta oficina participan o desarrollan los programas de la Red de Oficinas del SAE. En ella, trabajan actualmente dos dispositivos:

- Los técnicos dependientes del SEPE: se encargan de gestionar las prestaciones y los asuntos relacionados con los servicios públicos de empleo. En la oficina de empleo de Nerva trabajan dos técnicos en este servicio.

- Los técnicos dependientes del SAE: se encargan de proyectos diversos, y sus funciones y contratación depende de los mismos.

Los técnicos de esta red se relacionan, fundamentalmente, con los Ayuntamientos que componen la Cuenca Minera -para la gestión de ofertas de empleo, casi en exclusiva-, y con otras entidades que desarrollan programas de empleo formación. Han tenido cierta relación y coordinación con el personal integrante del programa "Andalucía Orienta". Por tanto, el nivel de coordinación y colaboración con otras entidades, resulta escaso.

Su dedicación a la gestión de proyectos y de ofertas de empleo hace que su influencia sobre el territorio sea muy limitada.

\subsection{Técnicos y personal de los CADE (Programa Andalucía Emprende)}

El Centro de Apoyo al Desarrollo Empresarial (CADE) es un instrumento público, que forma parte de la Red Territorial de Apoyo a Emprendedores de la Junta de Andalucía, que cuenta con 200 centros repartidos en 37 comarcas por el solar andaluz.

Existen dos sedes del CADE en la Cuenca Minera, una en Nerva y otra en Minas de Riotinto. Ambas pertenecen a la zona Andévalo (con un responsable técnico de zona), donde se incluyen también Valverde del Camino, Villanueva 
de las Cruces y Beas (fuera de la Cuenca Minera). En Minas de Riotinto existe un solo técnico y dos administrativos, mientras que Nerva cuenta con dos técnicos.

Las actividades y acciones que realizan en la Cuenca Minera, son las que les vienen determinadas por los servicios centrales de la Fundación Andalucía Emprende, como órgano responsable de su trabajo. Entre ellas se encuentran:

- Información y asesoramiento técnico cualificado a posibles emprendedores con intención de montar una empresa o realizar una actividad emprendedora. En ese caso se estudia el proyecto emprendedor, se analizan las posibilidades que pueda tener en el mercado, ayudándole a crear un plan de empresa viable.

- Impartición de formación en gestión empresarial básica y avanzada, según a que colectivos vaya dirigida.

- Tutorización en el proceso de creación de empresa, acompañando a los emprendedores en todo el proceso. No sólo en la creación sino también en el desarrollo, ayudando a consolidar su empresa.

- Búsqueda de vías de financiación, inversores o negocios con los que puedan cooperar para reforzar su posicionamiento en el mercado.

- Disposición de alojamiento empresarial, al objeto de facilitar espacio para que determinados emprendedores puedan instalarte y desarrollar su proyecto. En este sentido, son apoyados por técnicos especializados en creación y gestión de empresas, que ayudan a resolverle cualquier tipo de problema o duda.

- Participación en una serie de acciones provinciales, que se realizan puntualmente con el fin de dinamizar la cultura emprendedora local, adaptadas a las necesidades específicas de cada territorio. En este sentido se han realizado actividades de difusión, formación y encuentros del colectivo emprendedor a través de jornadas, eventos, talleres formativos, charlas, etc., donde se busca fomentar el espíritu emprendedor y empleador. Estas actividades se diseñan desde el propio territorio y en colaboración y trabajo en red con otros agentes territoriales. En los últimos años se han realizado actuaciones orientadas al turismo en Campofrío, a la artesanía en Nerva, al comercio en Minas de Riotinto y a nuevas oportunidades de negocio en Zalamea la Real, entre otras.

En lo referente, a la forma de atender a los diferentes emprendedores, las dos unidades CADE de la Cuenca Minera atienden indistintamente a cualquier emprendedor que los visite, sea cual sea su residencia o donde tenga pensado instalar la sede de su iniciativa. Una vez que comienzan a trabajar con él, lo tutorizan hasta el final, a no ser que él mismo quiera cambiar de técnico o de unidad por cualquier motivo. El emprendedor es quien decide.

De manera resumida, se puede decir que los técnicos CADE participan o desarrollan los siguientes programas de la Fundación Andalucía Emprende. 
- Promoción Cultura Emprendedora: Trabajan con colectivos del tipo de Escuelas Taller, Casas de Oficios Talleres de Empleo. También en institutos y en cursos de formación cuando se lo solicitan.

- En cuanto a los programas que desarrolla Fundación Andalucía Emprende en colaboración con la Consejería de Educación, y que engloba los programas denominados "Emprender en mi escuela", "Ícaro", "Empresa joven Europea", ó "Entrepreneurship Education", no se llevan a cabo en ningún colegio ni instituto de la C.M. Sus responsables no lo consideran prioridad en las enseñanzas que deben impartir.

- La iniciativa "Music Hero", si se ha puesto en marcha, con bastante buena acogida por parte de los jóvenes.

- En cuanto a la iniciativa "Emprende joven", se ha puesto en marcha en Nerva y Riotinto. No solo consiste en charlas de sensibilización, sino que el objetivo final es gestar una idea de negocio, con el fin de poder desarrollarla posteriormente. Han salido proyectos desarrollados en papel.

- En lo referente a los "Planes provinciales", donde se desarrollan jornadas se sensibilización en municipios concretos sobre sectores concretos, especiales para cada uno de ellos. Se han realizado actuaciones, orientadas al turismo en Campofrío, a la artesanía en Nerva, al comercio en Minas de Riotinto y a nuevas oportunidades de negocio en Zalamea la Real, entre otras. También existe cooperación internacional a través de "Transebt" con Portugal, desarrollando diferentes iniciativas cada año.

- En el "Día de la persona emprendedora", se invita a emprendedores locales a jornadas, seminarios, etc., que se diseñan y desarrollan en diferentes puntos de Andalucía. En el "Open Mind Awards", se transmite y anima a empresarios, emprendedores y empresas por si estuvieran interesados en participar. También existe un concurso para jóvenes con talento en Andalucía, donde desde Cuenca Minera se manda a jóvenes a participar.

- Desde el Plan Regional se desarrolla el programa "Senior" a través de expertos y "Lunar" apoyando la creatividad.

- También se lleva a cabo el llamado "Alojamiento empresarial", mediante el cual, una serie de dependencias - naves y despachos -, se prestan a empresas y emprendedores. Actualmente, se tienen cuatro despachos ocupados por emprendedores en Nerva y una nave por una empresa en Riotinto. En Riotinto se encuentra recién inaugurado un "Centro de apoyo al desarrollo empresarial", donde existen seis despachos para alojamiento empresarial, un patio de emprendedores y un lugar de gestión de recursos, reunión, etc. Actualmente tienen seis solicitudes para instalarse. 
Existe un ámbito de colaboraciones, a través de convenios con entidades como "La Caixa", "ICO", "Foram", etc., realizando funciones de agentes colaboradores.

Los técnicos están obligados a coordinarse con todos los agentes del territorio (ALPEs, ADR, Entidades financieras, Asociaciones de empresarios, etc.), no obstante, esa coordinación no siempre resulta fácil ni eficaz. Trataremos estos aspectos más adelante.

Existe otro apartado denominado "Análisis y prospectivas" donde se realizan estudios periódicos de ámbito socioeconómico, donde se aporta visión y apoyo para posibilidades futuras. Aquí no se realizan prospectivas, a pesar de la importancia de las mismas en un espacio con estas características.

\subsection{Técnicos y personal de la red de agentes de desarrollo local y promoción} de empleo (Consorcio UTEDLT Y ALPEs)

El programa de Unidades Territoriales de Empleo, Desarrollo Local y Tecnológico (UTEDLT) lo inicia en 2001 la Consejería de Empleo de la Junta de Andalucía en colaboración con las corporaciones locales, la Federación Andaluza de Municipios y Provincias (FAMP) y agentes económicos y sociales, dentro del marco del V Acuerdo de Concertación Social de Andalucía.

Están cofinanciadas por la Consejería y las corporaciones locales. La primera, asume el $100 \%$ de los costes de contratación de personal básico, equipos, mobiliario y equipamiento informático. En cuanto a Agentes Locales de Promoción de Empleo (ALPE), la Junta de Andalucía financia el 80\% de los costes laborales de estos. Las corporaciones locales se encargan de proporcionar los inmuebles y se hacen cargo de los gastos de funcionamiento.

En cuanto a la estructura organizativa de este dispositivo, hasta septiembre de 2012, fecha en que se disolvió esta red, estaba configurada por:

- Un Director UTEDLT, sin asignación de personal, pues este personal pasó a formar parte de los CADEs. Trabajaba en la sede de la Mancomunidad de Municipios Cuenca Minera, dependiendo jerárquica y funcionalmente de los servicios centrales de la Delegación de Empleo de Huelva.

- Una red conformada por seis ALPEs, con sede social y laboral en diferentes ayuntamientos de la Comarca (Berrocal, Campillo, Campofrío, Minas de Riotinto, Nerva y Zalamea la Real).

$\mathrm{Su}$ diseño y estructura, asentada en los territorios y orientadas a éstos, favorecía cierta complementariedad metodológica, enfocando e integrando bidireccionalmente los procesos de planificación y diseño anuales de sus intervenciones. Por un lado, implementando actuaciones sobre el territorio ligadas a la línea estratégica del Servicio Andaluz de Empleo -enfoque de arriba hacia abajo-, y, por otro, aquellas que desde los Consorcios se consideran necesarias 
como respuesta a las especificidades de los territorios -enfoque de abajo hacia arriba-.

Como ya se dijo previamente, tanto los Consorcios UTEDLT, como la red ALPEs se desmantelaron a finales de septiembre de 2012, a raíz de la crisis estructural y financiera. No obstante, hoy día, la mayoría de las funciones y competencias que tendía encomendada esta red (Unidad UTEDLT, junto a la red de ALPEs, de la Cuenca Minera), las ha asumido la red conformada por los CADE, perteneciente al programa "Andalucía Emprende".

Las actividades y acciones que realiza la unidad UTEDLT en la Cuenca Minera, son a grandes rasgos:

- Difusión. Comprenden desde realizar o colaborar en diversos eventos de mayor o menor relevancia, hasta los propios procesos de derivación e información que el SAE pone a disposición de la ciudadanía.

- Investigación. Al actuar en red con todos los dispositivos del territorio ha permitido obtener un conocimiento profundo de la realidad regional y provincial.

- Actividades enfocadas al diagnóstico de los territorios y a la actualización del conocimiento sobre los mismos. Asistencia técnica y captación de ofertas de empleo del SAE, atenciones y orientación sobre diferentes temáticas relacionadas -directa o indirectamente- con el empleo y el desarrollo local, a personas empleadoras, entidades y ciudadanía en general. Basados en que, un asesoramiento de calidad resulta un factor clave a la hora de generar empleo y crecimiento.

- Tramitación de documentación. Proceso continuo concerniente a la cumplimentación y entrega correspondiente de solicitudes de ayudas. Este servicio cesó, durante el año 2009, cuando se anuló el funcionamiento de los registros auxiliares de los Consorcios UTEDLT.

La misión principal de la red de ALPEs en la Cuenca Minera, son a grandes rasgos, la colaboración en acciones de fomento y promoción del desarrollo local en el ámbito municipal asignado. Entre sus funciones se encuentran:

- El análisis y la prospección del entorno socioeconómico.

- La promoción de proyectos y otras iniciativas de desarrollo local.

- Colaboración con los servicios del SAE.

- Dinamización y mejora de la competitividad de las PYMES en el territorio.

- Apoyo a la creación de empresas y promoción del autoempleo

- Colaboración con municipios y entidades del entorno para desarrollo de nuevos proyectos.

- Información, asesoramiento y recepción-entrega de documentación. Apoyo a la tramitación administrativa.

Desde el principio, los cambios sucesivos en la gestión de la red (paso de unas Consejerías a otras) y la dispersión entre instrumentos de sus competen- 
cias, llevó a la inoperancia del mismo, que fue concebido inicialmente como un instrumento de comarcalización administrativa de Andalucía, y nunca arrojó resultados.

\subsection{Técnicos y personal del GDR (Grupo de Desarrollo Rural): ADR Cuenca Minera}

En 1992, a través del Marco Comunitario 1992-1994, se constituye Ceder Cuenca Minera S.A., como Grupo de Desarrollo Rural para la gestión de la Iniciativa Comunitaria Leader I, siendo la primera actuación de desarrollo rural, propiamente dicha, de la Cuenca Minera. Fue creada como Grupo, por los siete Ayuntamientos que componen la comarca, para poder gestionar una ayuda pública puntual. Su sede se ubica, desde el principio, en el municipio de Minas de Riotinto.

La nueva Iniciativa Leader II, a través del Marco Comunitario 1995-1999, propicia el crecimiento de Ceder Cuenca Minera S.A., obligando al grupo de desarrollo a acceder a este nuevo Programa. De esta forma, se llevan a cabo importantes modificaciones en su estructura organizativa, quedando Homologado como Grupo de Desarrollo Rural, a los efectos del Plan de Desarrollo Rural de Andalucía, con la denominación: "Grupo de Desarrollo Rural de la Cuenca Minera de Huelva" y actuará como entidad colaboradora de la administración para la ejecución de Plan de Desarrollo Rural de Andalucía.

El 17 de marzo de 2000, bajo el nuevo Marco Comunitario 2000-2007, se constituye, con la denominación de Asociación para el Desarrollo Rural Cuenca Minera de Riotinto, la que será a partir de este momento el Grupo de Acción Local que gestione y dirija el Desarrollo Rural en la Cuenca Minera. Está formada por todas las entidades locales comarcales; entidades supramunicipales; sindicatos mayoritarios y patronal, asociaciones profesionales; empresas privadas; asociaciones de jóvenes y de mujeres; entidades bancarias; y Fundación Río Tinto, aglutinando en su seno toda la masa social de la Comarca Minera. De esta manera, se consigue que se constituya una sociedad de desarrollo, equilibrada entre lo público y lo privado, estructurada en torno a un órgano de gestión participativo y multisectorial, donde la mayoría de sus miembros, estaban representados en su Consejo de Administración.

En cuanto al marco financiero, del que obtiene sus fondos para actuar sobre el territorio, deriva del Fondo Europeo Agrícola de Desarrollo Rural (FEADER), en el ámbito del Nuevo Marco Comunitario de ayudas para el Desarrollo Rural en Andalucía y basado en el Reglamento (CE) 1698/2005 del Consejo, de 20 de septiembre de 2005. A través de éste, ADR pueden intervenir en su ámbito territorial ejecutando las estrategias de desarrollo, diseñadas y aplicadas por ellos, como el resto de Grupos de Desarrollo Rural (en adelante GDR) en sus respectivos ámbitos territoriales, siguiendo la metodología LEADER. 
Por lo que respecta a sus fines y objetivos, se encuentran:

1. Promover el desarrollo rural de los municipios de su ámbito de actuación.

2. Fomentar, facilitar, promocionar e impulsar toda clase de actividades generadoras de desarrollo económico y social, con cargo a recursos propios o a otras corporaciones, entidades, o administraciones públicas o privadas, especialmente aquellas que incidan en su ámbito territorial.

3. Desarrollo y gestión de iniciativas, programas, proyectos y convocatorias de las Administraciones Nacionales y Europeas, así como las promovidas por entidades privadas.

4. Promover programas de formación y empleo, en cualquiera de sus modalidades, que favorezca el desarrollo y la integración de todos los colectivos de la comarca.

5. Favorecer el desarrollo endógeno y sostenido de la zona través de la diversificación económica, con especial atención en la preservación del medioambiente y el patrimonio.

6. Colaborar con otras entidades con fines análogos para el desarrollo de programas comunes.

7. Impulsar la investigación en materia de desarrollo rural y medio ambiente, en colaboración con la Universidad y otros centros.

8. Recepción, gestión, estudio y financiación con cargo a recursos propios o ajenos de cuantos proyectos se consideren viables.

9. Propiciar la integración del enfoque de género y juventud.

10. La gestión de todo tipo de ayudas y actividades destinadas a financiar la Asociación y a la creación de la infraestructura necesaria para garantizar su funcionamiento.

A raíz del inicio del nuevo Marco Comunitario 2000-2007, se abre un nuevo panorama en el Desarrollo Rural Andaluz, marcado por dos hitos clave:

1. El 29 de diciembre de 2000 la Comisión Europea aprueba el Programa Operativo Integrado de Andalucía (Decisión C (2000) 39565) que contiene, de conformidad con lo establecido en el R. CE 1260/1999, de 21 de junio, las medidas propuestas y un plan de financiación para el desarrollo del Eje $7^{\circ}$ "Agricultura y Desarrollo Rural", que contiene las Medidas 7.55 y 7.59: Desarrollo Endógeno de Zonas Rurales ligadas a actividades No Agrarias. Situación que supone un importante apoyo institucional por parte de la Junta de Andalucía.

2. El Decreto $7 / 2000$, de 15 de enero, por el que se regula la participación en la gestión del Programa de Desarrollo Endógeno de Zonas Rurales de Andalucía (PRODER), cuya gestión y ejecución se realizará según lo dispuesto en el art. 2.1 con la colaboración de las Asociaciones de Desarrollo Rural. Lo que viene a suponer la creación de un Modelo de Desarrollo Rural, hecho a medida para Andalucía, basado en el trabajo 
que vienen realizando los 49 Grupos de Desarrollo Rural andaluces. Modelo que, como hemos visto, se convertirá en un referente para el resto del país.

ADR Cuenca Minera, fue seleccionada para colaborar en la gestión del Proder-A, manteniendo la misma filosofía de trabajo para el Desarrollo de la Comarca, aunque bajo una forma jurídica diferente. Desde entonces se han desarrollado un conjunto de trabajos entre los que cabe destacar:

1. Elaboración de un Plan Estratégico Básico para la Cuenca Minera de Riotinto.

2. Colaboración en la ejecución de las políticas de desarrollo rural de la Comunidad Autónoma mediante la ayuda a emprendedores.

3. Incorporar la Perspectiva de Género y Juventud en las Actuaciones de Desarrollo Rural.

4. La gestión del Programa de Desarrollo Rural PRODER-A.

5. La gestión del proyecto FILÓN, desarrollado al amparo de la Iniciativa Comunitaria Equal y cofinanciado por el Fondo Social Europeo y la Consejería de Empleo de la Junta de Andalucía.

6. La gestión del proyecto IDE@ enmarcado y desarrollado al amparo de la Iniciativa Comunitaria Equal y cofinanciado por el Fondo Social Europeo y la Consejería de Empleo de la Junta de Andalucía.

7. Puesta en marcha, en colaboración con el Grupo de Desarrollo de la Comarca Sevillana del Corredor de la Plata, del Plan de Turismo Sostenible para la Faja Pirítica del Suroeste Peninsular.

8. Coordinación de la Acción Conjunta "Aprovechamiento Integral de los Espacios Mineros Degradados", junto a otras ocho comarcas mineras de Andalucía.

9. Participación y miembro activo del Grupo de Cooperación "Espacios Degradados de Andalucía".

En la actualidad, la Asociación para el Desarrollo Rural de la Cuenca Minera, está inmersa en el nuevo Marco Comunitario 2007-2013, por el que, a través del Nuevo Programa de Desarrollo Rural de Andalucía 2007-2013, diseña y comienza a aplicar una Estrategia Comarcal, adaptándola de forma transversal y bajo el enfoque Leader, creando un Plan de actuación para la Cuenca Minera, con intervenciones directas agrupadas en Líneas de Intervención; Subprogramas y Programas, en consonancia con lo dispuesto en el Reglamento (CE) $\mathrm{n}^{\circ}$ 1698/2005, de FEADER. A través de este Plan de actuación, va a desarrollar una serie de medidas destinadas a la conservación del medio ambiente, la diversificación de la economía y la mejora de la calidad de vida de los territorios rurales. 
3.5. Técnicos y personal de Asociaciones, Fundaciones, Sindicatos, Asociaciones de empresarios, etc., que contribuyen en el desarrollo Comarcal

Existen otros instrumentos de fomento del empleo de carácter privado, aunque dependen de fondos públicos, retraídos en la actualidad.

Se trata de un conjunto de entidades de muy diferente naturaleza y con fines muy diversos, con un vínculo, al menos, en común. Todas estas estructuras o entidades interactúan con el entorno en tareas sociales o labores relacionadas, en mayor o menor grado, con la promoción del desarrollo económico y social en la comarca, en función a sus posibilidades o a sus ámbitos de actuación. Dependiendo del tipo de entidades sus objetivos o fines son muy diversos. Hay entidades que persiguen la integración de los disminuidos físicos o psíquicos (dependientes), otras ayudan a los familiares y enfermos de algunas enfermedades degenerativas (Alzheimer, demencia senil, etc.), rehabilitan el patrimonio minero con fines turísticos como vía alternativa de desarrollo, facilitan la financiación de proyectos, apoyan las necesidades de los empresarios, o las de los trabajadores, etc. Como ejemplo, tenemos:

1. Entidades que trabajan en pro de la integración de determinados colectivos: Aspromin (Asociación Protectora de Personas con Discapacidad Intelectual de la Cuenca Minera), AFA (Asociación de familiares de enfermos de Alzheimer), Athenea (Asociación de personas con discapacidad), entre otras.

2. Estas entidades actúan sobre el territorio ayudando a personas dependientes, mejorando el nivel y la calidad de vida, de éstos, y de sus familiares. Trabajan aspectos como la formación adaptada para estos colectivos y su posterior inserción profesional. El personal que trabaja en este ámbito está formado por gestores de la salud, formadores y personal sanitario, entre otros.

3. Entidades que trabajan en pro de la rehabilitación del patrimonio histórico, natural y/o cultural con diferentes fines: Fundación Río Tinto (para la historia de la minería y la metalurgia), Asociación Amigos del Ferrocarril, Asociación para la Defensa del Patrimonio de Zalamea la Real, Asociación Cistus Jara, etc.

4. Estas entidades actúan sobre el territorio defendiendo su Patrimonio, en sus múltiples facetas, rehabilitándolo, conservándolo, difundiéndolo, etc. Trabajan aspectos como la formación, divulgación, rehabilitación y conservación de Patrimonio Arquitectónico, natural, cultural, etc. El personal que trabaja en este ámbito está formado por gestores culturales, formadores y personal técnico especializado -arquitectos, ingenieros, biólogos, arqueólogos e historiadores, entre otros.

5. Entidades de ahorro o crediticias: formadas por Oficinas Bancarias o Cajas de Ahorro. Actúan sobre el territorio apoyando financieramente 
Proyectos de muy diversa naturaleza, favoreciendo el flujo económico sobre el territorio, al gestionar operaciones financieras de personas físicas y jurídicas. El personal que trabaja en este ámbito, está formado por gestores económicos fundamentalmente.

6. Entidades que trabajan en pro de los derechos de los trabajadores: formadas por organizaciones sindicales representadas en la Cuenca Minera como, Comisiones Obreras (CCOO), Unión General de Trabajadores (UGT) o la Central Sindical Independiente y de Funcionarios. Trabajan aspectos como la supervisión salarial de los trabajadores, sus condiciones laborales y la mejora de la formación de éstos. El personal que trabaja en este ámbito suele estar formado por trabajadores, de muy diferente formación, liberados o no, de empresas de la Comarca.

7. Entidades que trabajan en pro de las empresas, empresarios y demás sociedades: formadas por asociaciones de empresarios, representadas en la Cuenca Minera por ACIR (Asociación de pequeños comerciantes e industriales de Riotinto) y por APYME (Asociación local de empresarios miembros de la Federación Onubense de Empresarios). Dirigen su trabajo hacia las pymes y autónomos de la localidad, promoviendo actividades de cara a fomentar el autoempleo en la zona, sacando el mayor partido posible a las posibilidades que brinda la zona a otros sectores como la industria auxiliar y transformadora, la agricultura, etc. poniendo en sus manos instrumentos que relaciones a las empresas con la sociedad de la información, telecomunicaciones y nuevas tecnologías; internacionalización, etc. El personal que trabaja en este ámbito suele estar formado por los propios empresarios del territorio.

8. Otras Asociaciones que trabajan en pro de determinados colectivos: formadas por asociaciones de mujeres y jóvenes, fundamentalmente. Persiguen diferentes objetivos y tienen intereses muy variados.

\section{Conclusiones}

Desde las administraciones públicas, a los tres niveles escalares, se ha dado un intento de crear estructuras de desarrollo en la Comarca de la Cuenca Minera, con una crisis estructural y condiciones territoriales especiales vinculadas con el monocultivo minero.

La creación e implementación de instrumentos de desarrollo y empleo (empleabilidad) ha sido muy importante, y sobre ellas se puede concluir:

- Las soluciones propuestas para la recuperación económica de la comarca y el crecimiento del empleo pasa de nuevo por la reapertura de las minas, una vez recuperados los márgenes de rentabilidad.

- Los instrumentos privados han conseguido la generación de más desarrollo y empleo que los públicos, si bien es cierto que están subsidiados 
o necesitan de las ayudas públicas para su mantenimiento (por su carácter no productivo).

- Las directrices políticas, marcadas desde la administración autonómica ha hecho que algunos de los instrumentos hayan tenido un nulo efecto sobre la comarca.

- El desmantelamiento de estos instrumentos demuestra que, en muchos casos, lo que se fomentó desde la administración fue la creación de estructuras de desarrollo (empleo técnico) y no el verdadero fomento del empleo a partir de la mejora de las estructuras productivas y creación de sinergias.

- La superposición de estructuras de desarrollo invalidó el funcionamiento de las mismas por el choque de competencias y la diversificación de actuaciones, en ocasiones, desvinculadas de la realidad productiva del territorio.

- La estructura propuesta de comarcas, a veces rota por algunos de los instrumentos, coincide con un intento de establecer "comarcas políticamente construidas", que no responden a la realidad territorial y, por tanto, no atiende a las necesidades reales de la población.

El contexto de crisis actual debe ver las oportunidades que se abren en el territorio. La reapertura de las minas debe suponer la creación de una estructura de empleo paralela, pero no dependiente de la actividad minera, sino capaz de sustraerse de esta, al tiempo que se beneficia de la afluencia de capitales. Para ello, las administraciones públicas y entes privados deben aunar esfuerzos y sintetizar las estructuras de desarrollo, empleo y empleabilidad, unificando líneas de actuación, proponiendo soluciones viables desde abajo y evitando la multiplicación de entes innecesariamente.

\section{BibliOgRAFÍA Y FUENTES DOCUMENTALES}

BENITO DEL POZO, P. (2002): "Patrimonio industrial y cultura del territorio", Boletín de la AGE, $\mathrm{n}^{\circ} 34$, pp. 213-227.

CABELLO LÓPEZ, F. J. (2011): "Programas formativos de empleo en la rehabilitación del patrimonio industrial", en PÉREZ MACÍAS, J. A. et al. (2011). Rio tinto: Historia, patrimonio minero y turismo cultural. Fundación Río Tinto, Universidad de Huelva. Huelva, pp. 595-618.

CABELLO LÓPEZ, F. J. (2012): "ESTRUCTURAS PROMOTORAS DEL DESARROLLO EN LA CUENCA MINERA DE RIOTINTO: Papel e incidencia sobre su realidad socioeconómica. Estado de la cuestión. Propuestas para su mejora", Trabajo Fin de Máster ESTEAM, Universidad de Huelva. Inédito.

FORONDA, C. y JOYA, M. P. (2005): "Características del tejido empresarial en la Cuenca Minera de Riotinto (Andalucía)", en Manero, F. y Pascual, 
H. (Coords.): Innovación tecnológica, servicios a las empresas y desarrollo territorial, Universidad de Valladolid, Valladolid, pp. 293-308.

GARCÍA-DELGADO, F. J., DELGADO-DOMÍNGUEZ, A. Y FELICIDADES-GARCÍA, J. (2013): "El turismo en la Cuenca Minera de Riotinto", en Cuadernos de Turismo, no 31, pp. 129-152.

LLURDÉS I COIT, J. C. et al. (1999): "Conflictos locacionales en territorios en crisis. Turismo y residuos en Cardona (Barcelona)", en Anales de Geografía de la Universidad Complutense, $\mathrm{n}^{\circ}$ 19, pp. 119-140.

MÁRQUEZ DOMÍNGUEZ, J. A. (Dir.) (2002): Plan Estratégico de Desarrollo Rural para el Andévalo Occidental, Instituto de Desarrollo Local y Consejería de Educación y Ciencia de la Junta de Andalucía, San Juan del Puerto.

VALENZUELA RUBIO, M. et al. (2008): "La valorización turística del patrimonio minero en entornos rurales desfavorecidos. Actores y experiencias", Cuadernos de Turismo, $\mathrm{n}^{\circ}$ 22, pp. 231-260. 